\title{
Negative inductance circuits for metamaterial bandwidth enhancement
}

\author{
Emilie Avignon-Meseldzija ${ }^{1,3}$, Thomas Lepetit ${ }^{2}$, Pietro Maris Ferreira ${ }^{1}$, and Fabrice Boust ${ }^{2,3, *}$ \\ ${ }^{1}$ GeePs, UMR CNRS 8507, CentraleSupélec, Université Paris-Saclay, 91192 Gif-sur-Yvette, France \\ 2 ONERA - The French Aerospace Lab, 91120 Palaiseau, France \\ ${ }^{3}$ SONDRA, CentraleSupélec, Université Paris-Saclay, 91192 Gif-sur-Yvette, France
}

Received: 3 June 2017 / Received in final form: 19 October 2017 / Accepted: 27 October 2017

\begin{abstract}
Passive metamaterials have yet to be translated into applications on a large scale due in large part to their limited bandwidth. To overcome this limitation many authors have suggested coupling metamaterials to non-Foster circuits. However, up to now, the number of convincing demonstrations based on non-Foster metamaterials has been very limited. This paper intends to clarify why progress has been so slow, i.e., the fundamental difficulty in making a truly broadband and efficient non-Foster metamaterial. To this end, we consider two families of metamaterials, namely Artificial Magnetic Media and Artificial Magnetic Conductors. In both cases, it turns out that bandwidth enhancement requires negative inductance with almost zero resistance. To estimate bandwidth enhancement with actual non-Foster circuits, we consider two classes of such circuits, namely Linvill and gyrator. The issue of stability being critical, both metamaterial families are studied with equivalent circuits that include advanced models of these non-Foster circuits. Conclusions are different for Artificial Magnetic Media coupled to Linvill circuits and Artificial Magnetic Conductors coupled to gyrator circuits. In the first case, requirements for bandwidth enhancement and stability are very hard to meet simultaneously whereas, in the second case, an adjustment of the transistor gain does significantly increase bandwidth.
\end{abstract}

Keywords: Metamaterials / Non-Foster circuit / Linvill / Gyrator / Artificial magnetic media / Artificial magnetic conductor

\section{Introduction}

Ever since the turn of the millennium, metamaterials have paved the way for very innovative and promising propositions. However, in most early demonstrations, the useful frequency bandwidth was quite limited and that significantly hindered the translation of metamaterials concepts into industrial applications. Quickly, it was realized that these narrow bandwidths were inherently linked to the linear and passive, thus causal, nature of metamaterials [1-3]. Following this observation, several authors suggested to couple metamaterials with nonFoster circuits to overcome these limitations [4-11].

Foster's theorem states that the reactance of a passive, lossless, two-terminal network strictly increases with frequency [12]. Any circuit that violates this rule is thus referred to as non-Foster (NF). Usually, NF circuits aim to generate pure negative reactances (negative capacitances, inductances, or both), i.e., with the least amount of

\footnotetext{
* e-mail: fabrice.boust@onera.fr
}

resistance. On one hand, NF circuits have been successfully used to improve impedance matching of small antennas, by introducing a negative capacitance in the feeding network [13-20]. On the other hand, the number of NF circuits that demonstrated significant metamaterial bandwidth enhancement with a negative inductance is still very limited $[20,21]$, despite many proposals $[5-8,11]$. This paper thus focuses only on negative inductance circuits among all nonFoster circuits.

This paper intends to clarify the fundamental difficulty in making a truly broadband and efficient NF metamaterial. To this end, we consider two families of metamaterials: Artificial Magnetic Media (AMM) and Artificial Magnetic Conductors (AMC). These two families have been extensively studied, yet still hold a great potential for innovative applications. In both cases, bandwidth enhancement requires a non-Foster impedance able to compensate the intrinsic positive inductance with as little resistance as possible.

This paper is organized in four parts. In the first one, we present both AMMs and AMCs, highlighting in both cases the need for NF circuits. In the second one, we introduce 


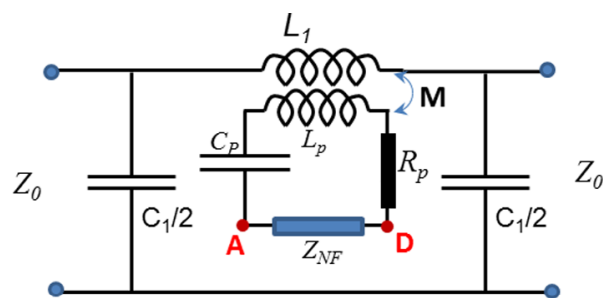

Fig. 1. Equivalent circuit modeling a SRR array: PI network of a transmission line connected, via a mutual inductance, to a RLC series resonant circuit. ( $L_{p}$ : loop inductor, $C_{p}$ : loop + lumped capacitor, $Z_{\mathrm{NF}}$ : NF impedance, $R_{p}$ : loop resistance).

two types of NF circuits, namely the Linvill and gyrator, and discuss their performances. In the third one, we discuss the critical issue of NF circuits stability, both unloaded and loaded, coupling AMMs to Linvills and AMCs to gyrators. In the fourth one, we assess performances of both NF circuits in their stable configuration and explain their respective limitations. This is followed by a conclusion and prospects.

\section{Non-Foster AMM and AMC}

\subsection{Non-Foster AMM}

AMMs are essentially arrays of resonators with a non-zero magnetic dipole moment [22]. At resonance, the structure behaves like an effective medium with a dispersive non-zero magnetic permeability. Hence, AMMs are a leading choice in magneto-dielectric antenna substrates and MRI lenses $[23,24]$. However, like all metamaterials, AMMs bandwidth is quite limited. In 2013, a collaboration from Northeastern and Cornell universities was the first to show that a NF negative inductor can significantly increase the operating bandwidth of an AMM [8].

In this paper, we consider a specific AMM, namely an array of split-ring resonators (SRRs). A single SRR can be modeled as a simple RLC series resonant circuit [25], while an array of SRRs can be modeled as a Transmission Line (TL) connected, via a mutual inductance, to a RLC series resonant circuit. Furthermore, a lumped capacitor and a NF circuit can be introduced (Fig. 1).

In the following, we consider a NF circuit generating a negative inductance $\left(Z_{\mathrm{NF}}=R_{\mathrm{NF}}+j L_{\mathrm{neg}} \omega\right)$. Due to its RLC series character, this AMM has the following resonance frequency:

$$
\begin{aligned}
& \omega_{o}=1 / \sqrt{L_{t} C_{p}} \\
& \text { with } L_{t}=L_{p}+L_{\text {neg }}
\end{aligned} .
$$

Besides, the bandwidth at half-maximum is given by:

$$
\begin{aligned}
& \Delta \omega=R_{t} C_{p} \omega_{o}^{2}=R_{t} / L_{t} \\
& \text { with } R_{t}=R_{p}+R_{\mathrm{NF}}
\end{aligned} .
$$

Obviously, it becomes infinitely large when $L_{t}$ tends towards zero, i.e., when $L_{\text {neg }}$ tends towards $-L_{p}$. Thus, to simultaneously increase the bandwidth and keep the resonance frequency constant, we need to increase the capacitance $C_{p}$.

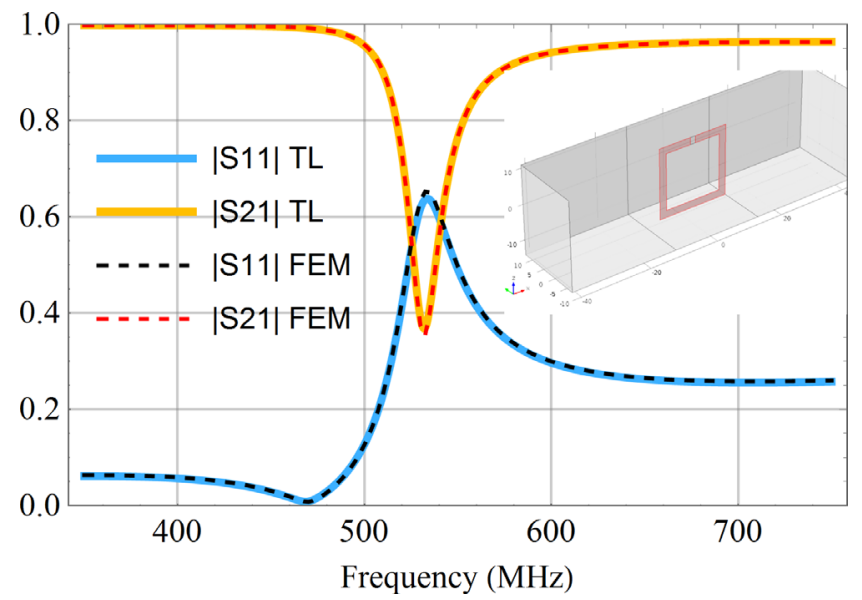

Fig. 2. Comparison of S-parameters calculated by full-wave simulations with the equivalent circuit of Figure $1\left(C_{1}=0.2 \mathrm{pF}\right.$, $L_{1}=31.5 \mathrm{nH}, M=18.5 \mathrm{nH}, L_{p}=45 \mathrm{nH}, C_{p}=2 \mathrm{pF}$ and $R_{p}=3 \Omega$ ). Insert: Geometry of the calculated SRR. Cell size: $25 \times 20 \times 25 \mathrm{~mm}$. SRR length: $20 \mathrm{~mm}$, SRR strip width: $2 \mathrm{~mm}$, SRR gap: $1.5 \mathrm{~mm}$. Periodic boundary conditions are assumed in both lateral directions.

Full-wave simulations, using the Finite-Element Method (FEM), were run to estimate the accuracy of our equivalent circuit. Results clearly demonstrate its validity (Figure 2). Hence, we ran several equivalent circuit simulations, varying both $L_{\text {neg }}$ and $R_{\mathrm{NF}}$, assuming that neither of these parameters depends on frequency, while adjusting $C_{p}$ to keep the resonance frequency constant.

From S-parameters, permittivity and permeability were obtained with the NRW algorithm [26,27]. It is well known that this algorithm can lead to a permittivity with a non-physical meaning near the resonance. Some authors have proposed new algorithms to circumvent this difficulty [28] but, if the permittivity is then quite different, the permeability does remain equal to that given by the NRW algorithm. Thus, we still use it in the following.

Figure 3 illustrates the two main impacts of the NF impedance introduction into the circuit. First, that of a pure negative inductance $\left(L_{\text {neg }}=-40 \mathrm{nH}\right)$, which almost compensates the loop inductance, and, second, that of an additional resistance $\left(R_{\mathrm{NF}}=17 \Omega\right)$, which stems from the actual NF impedance.

From this short study, two important lessons can be drawn:

- A perfect negative inductance substantially increases the bandwidth corresponding to a useful permeability, provided $\left|L_{\text {neg }}\right|$ is close to $L_{p}$ (with $\left|L_{\text {neg }}\right|<L_{p}$ for stability, Section 4.2).

- The resistive part associated to the NF impedance should not exceed a few ohms to effectively obtain a large bandwidth.

\subsection{Non-Foster AMC}

AMCs are essentially capacitive Frequency Selective Surfaces (FSS) on a ground plane [29]. At resonance, an incident wave is reflected without any phase shift and the structure behaves as a perfect magnetic conductor. Hence, 


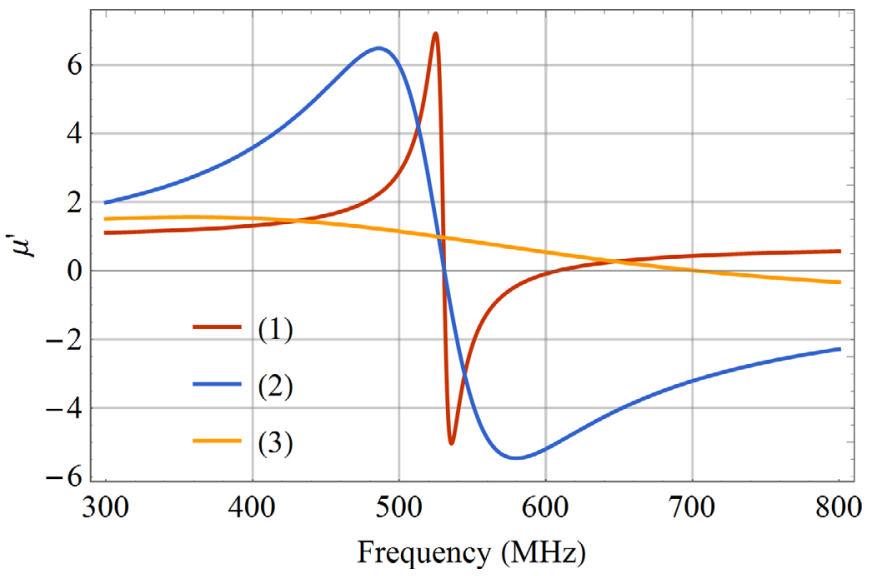

Fig. 3. Effective permeability (real part) of a SRR array vs frequency: (1) $L_{\mathrm{neg}}=0, C_{p}=2 \mathrm{pF}, R_{\mathrm{NF}}=0 \Omega(2) L_{\mathrm{neg}}=-40 \mathrm{nH}$, $C_{p}=18 \mathrm{pF}, R_{\mathrm{NF}}=0 \Omega(3) L_{\mathrm{neg}}=-40 \mathrm{nH}, C_{p}=18 \mathrm{pF}, R_{\mathrm{NF}}=17 \Omega$.

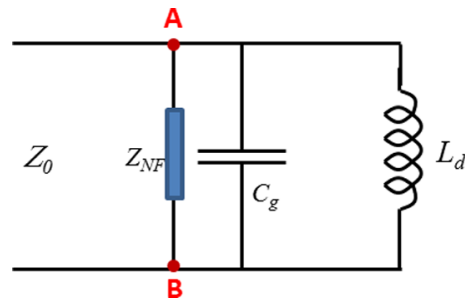

Fig. 4. Equivalent circuit modeling a patch array: shortcircuited transmission line connected to a capacitance, thus forming a LC series resonant circuit. ( $L_{d}$ : short-circuit inductor, $C_{g}$ : patch + lumped capacitor, $Z_{\mathrm{NF}}$ : NF impedance).

AMCs are a leading choice for low-profile antennas or thin radar-absorbing materials [30-32]. AMCs are especially attractive at low operating frequencies due to their extremely small thickness. However, like all metamaterials, AMCs bandwidth is quite limited. In 2011, a group from HRL Laboratories was the first to show that a NF negative inductor can significantly increase the operating bandwidth of an AMC [21].

In this paper, we consider a specific AMC, namely a patch array placed at a distance $d$ above a ground plane. When $d$ is small compared to $\lambda$, the propagation between the FSS and the ground plane is equivalent to an inductance $L_{d}=\mu_{0} d$. Thus, an AMC is equivalent to a LC parallel resonant circuit (Fig. 4).

In the following, we consider a NF circuit generating a negative inductance $\left(Z_{\mathrm{NF}}=R_{\mathrm{NF}}+j L_{\mathrm{neg}} \omega\right)$. Due to its LC parallel character, this AMC has the following resonance frequency:

$$
\begin{aligned}
& \omega_{o}=1 / \sqrt{L_{t} C_{g}} \\
& \text { with } L_{t}=\left(\frac{1}{L_{p}}+\frac{1}{L_{\text {neg }}}\right)^{-1} .
\end{aligned}
$$

Besides, the derivative of the phase shift on resonance, which is the relevant figure of merit, is given by:

$$
\left(\frac{d \phi}{d \omega}\right)_{\omega_{0}}=-4 Z_{0} C_{g}=-4 Z_{0} /\left(\omega_{0}^{2} L_{t}\right)
$$

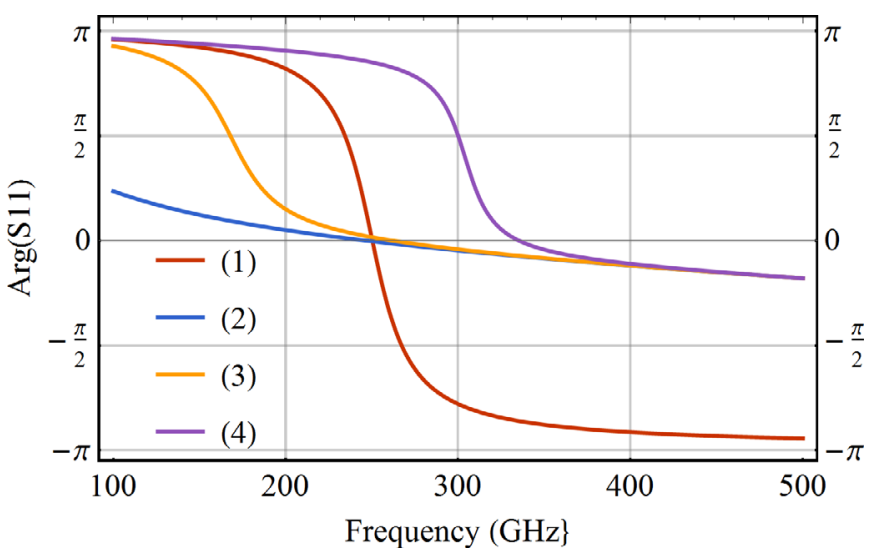

Fig. 5. Phase on reflection of a patch array vs frequency: (1) No negative inductance, $C_{g}=C_{\mathrm{gl}}=12.8 \mathrm{pF}(2) L_{\mathrm{neg}}=-32.2 \mathrm{nH}, C_{g}=$ $C_{\mathrm{g} 0}=0.25 \mathrm{pF}, R_{\mathrm{NF}} 0 \Omega(3) L_{\mathrm{neg}}=-32.2 \mathrm{nH}, C_{g}=C_{\mathrm{g} 0}=0.25 \mathrm{pF}$, $R_{\mathrm{NF}}=3 \Omega(4) L_{\mathrm{neg}}=-32.2 \mathrm{nH}, C_{g}=C_{\mathrm{g} 0}=0.25 \mathrm{pF}, R_{\mathrm{NF}}=10 \Omega$.

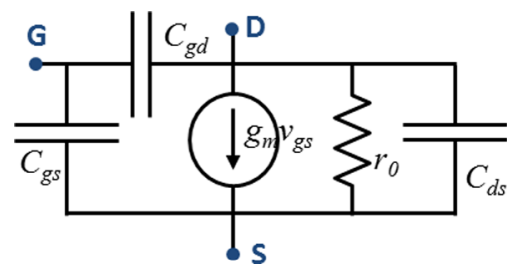

Fig. 6. Small signal model of the MOSFET [37]. The three nodes correspond to the Gate $(G)$, Source $(S)$, and Drain (D).

Obviously, it becomes infinitely small when $L_{t}$ tends towards infinity, i.e., when $L_{\text {neg }}$ tends towards $-L_{d}$. Thus, unlike the AMM, to simultaneously increase the bandwidth and keep the resonance frequency constant, we need to decrease the capacitance $C_{g}$.

As for the AMM, full-wave simulations were run to estimate the accuracy of our equivalent circuit. Results clearly demonstrate its validity (not shown). Hence, we ran several equivalent circuit simulations, varying both $L_{\text {neg }}$ and $R_{\mathrm{NF}}$, assuming that neither of these parameters depends on frequency, while adjusting $C_{g}$ to keep the resonance frequency constant.

We have considered a patch array placed at $d=25 \mathrm{~mm}$ over a ground plane $\left(L_{d}=31.4 \mathrm{nH}\right)$, with a small patch capacitance $(0.25 \mathrm{pF})$. With these parameters, the structure behaves as an AMC around 1.6 GHz. Since our initial working frequency is too high, we have two options to lower it down to $250 \mathrm{MHz}$. We can either add a lumped capacitor to increase $C_{g}$ (up to $12.8 \mathrm{pF}$ ) or add a negative inductor to increase $L_{t}$ (up to $1290 \mathrm{nH}$ for $L_{\mathrm{neg}}=-32.2 \mathrm{nH}$ ).

Figure 5 illustrates the two main impacts of the NF impedance introduction into the circuit. First, that of a pure negative inductance $\left(L_{\mathrm{neg}}=-32.2 \mathrm{nH}\right)$, which almost compensates the loop inductance, and, second, that of an additional resistance $\left(R_{\mathrm{NF}}=3\right.$ or $\left.10 \Omega\right)$, which stems from the actual NF impedance.

From this short study, two important lessons can be drawn:

- A perfect negative inductance substantially increases the bandwidth corresponding to a PMC behavior, provided $\left|L_{\text {neg }}\right|$ is close to $L_{d}$ (with $\left|L_{\text {neg }}\right|>L_{d}$ for stability, Section 4.4). 
- The resistive part associated to the NF impedance should not exceed a few ohms to effectively obtain a large bandwidth.

In summary, both AMMs and AMCs require a negative inductance with a small resistive part. Hence, some of the requirements placed on NF circuits are common to these two structures. However, one major difference is their series versus parallel character. This has dramatic consequences on the choice of a relevant NF architecture as discussed next.

\section{Negative inductance circuits}

Among all possible circuit architectures that can generate negative inductances, two have been selected: Linvill (a Negative Impedance Converter or NIC: $Z \rightarrow-Z$ ) and gyrator (a Negative Impedance Inverter or NII: $Z \rightarrow-1$ / $Z)$. A majority of architectures proposed in the literature are based on these two.

Many authors pointed out the negative impact of transmission lines and transistor packages on Non-Foster circuits [33-36]. As Loncar et al. have shown, transmission lines have an impact on both reactance and resistance. Thus, the electrical length of transmission lines should be reduced as much as possible. Consequently, we have decided to work with integrated circuits.

We chose to base both topologies on MOSFET (Metal Oxide Semiconductor Field Effect Transistor), which is the most commonly used transistor in microelectronics. The classical small signal model of the MOSFET, presented in Figure 6, is used in the following [37].

In Figure $6, g_{m}$ is the transistor transconductance, $v_{\mathrm{gs}}$ its gate-source voltage, $r_{0}$ its output resistance (with or without a drain resistor in parallel), and $C_{\mathrm{gs}}, C_{\mathrm{gd}}, C_{\mathrm{ds}}$ its parasitic capacitances. Values of the above parameters depend on technology, bias point, and transistor dimensions. For instance, larger transistors have larger parasitic capacitances. We chose to consider a mature and readily accessible technology (CMOS $0.35 \mu \mathrm{m})$. Following values remain constant throughout the paper: $C_{\mathrm{par}}=C_{\mathrm{gs}}+C_{\mathrm{ds}}=$ $0.4 \mathrm{pF}, C_{\mathrm{gd}}=0.2 \mathrm{pF}, r_{0}=2 \mathrm{k} \Omega$.

\subsection{Negative inductance based on Linvill circuits}

The principle of the Linvill circuit was first published in [38]. Since then, a large number of implementations have been proposed, some using discrete components [15,39-41], and others fully integrated $[42,43]$. Since it is a NIC, it provides negative inductance/capacitance when loaded with an inductor/capacitor.

Figure $7 \mathrm{a}$ presents the principle of the Linvill circuit while Figure 7b presents its small-signal model. Considering a simplified model, without parasitic capacitors, the input impedance is given by:

$$
Z_{\text {Linv }}=\frac{2 r_{0}}{g_{m} r_{0}+1}-Z_{L} \frac{g_{m} r_{0}-1}{g_{m} r_{0}+1} \approx \frac{2}{g_{m}}-Z_{L} .
$$

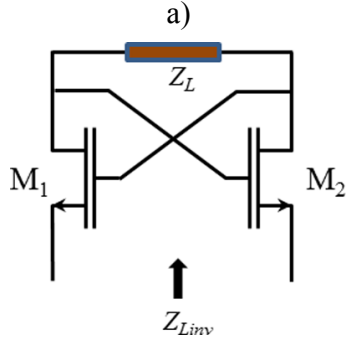

b)

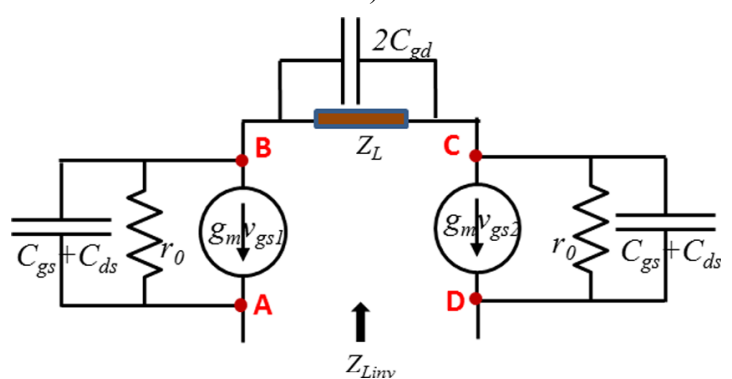

Fig. 7. a) Linvill circuit with MOSFET transistors. b) Smallsignal model of the Linvill circuit.

The final approximation was obtained by assuming $g_{m} r_{0}>>1$. Thus, for the Linvill, the reactive part of the impedance only depends on the load and the resistive part is positive and decreases with $g_{m}$.

At first glance, it would then seem as though one only need consider a pure inductance $\left(Z_{L}=j L \omega\right)$ together with a large $g_{m}$. However, a large $g_{m}$ implies large power consumption, as shown in [42] where $g_{m}$ has the unusually large value of $30 \mathrm{mS}$. A practical alternative is to add a compensation resistor in series $\left(Z_{L}=R+j L \omega\right)$ to decrease the resistive part. In the following, we consider the full model, i.e., including compensation resistor, parasitic capacitors and without any approximation (for the complete expression of the input impedance see Appendix A.1).

In the Linvill circuit, two parameters are readily controllable: $g_{m}$ and $R$. Even within the full model, they afford enough degrees of freedom to cancel the real part of the NF impedance at any frequency of interest. To illustrate this fact, we consider a specific example $\left(g_{m}\right.$ is limited to $20 \mathrm{mS}$ in order to consider achievable values). Figure 8 presents results that were obtained with $R$ varying from $0 \Omega$ to $100 \Omega\left(g_{m}=20 \mathrm{mS}, L=40 \mathrm{nH}\right)$. We see that the real part always cancels at some frequency $\left(f_{\mathrm{Re} 0}\right)$, which increases with $R$. Besides, we observe that the real part quickly takes on large negative values beyond $f_{\mathrm{Re} 0}$.

Conclusions of this section are threefold. First, a negative inductance can be obtained by introducing an inductor in the load impedance. Second, cancellation of the real part can be achieved either by a large $g_{m}$ or by adding a resistor $R$ to the load impedance, and this can be achieved at any frequency of interest. Finally, minimization of the real part on a large bandwidth cannot be done around any frequency but, rather, is achievable only at low frequencies $(f<200 \mathrm{MHz})$ and for large compensation resistor $(R>100 \Omega)$. 

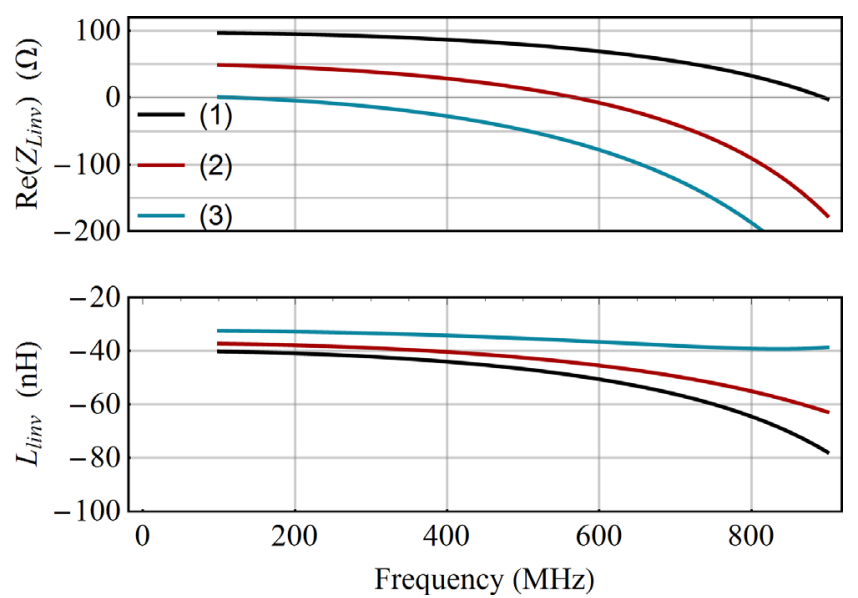

Fig. 8. Input impedance of the Linvill circuit, $Z_{\mathrm{Linv}}=R e$ $\left(Z_{\text {Linv }}\right)+j L_{\text {Linv }} \omega$, assuming $g_{\mathrm{m}}=20 \mathrm{mS}, \quad L=40 \mathrm{nH}$ and: $(1)$ $R=0 \Omega, \quad f_{\operatorname{Re} 0}=900 \mathrm{MHz} \quad(2) \quad R=50 \Omega, \quad f_{\operatorname{Re} 0}=570 \mathrm{MHz} ; \quad(3)$ $R=100 \Omega, f_{\operatorname{Re} 0}=120 \mathrm{MHz}$.

\subsection{Negative inductance based on Gyrator circuits}

Gyrator circuits [44,45] have been massively employed for IC implementation of active inductors to avoid integration of passive spiral inductors [46], which take up a lot of space. Recently, this topology has even been used in an IC to obtain a negative inductance dedicated to AMCs [47]. Since it is a NII (Negative Impedance Inverter), it provides negative inductance /capacitance when it is loaded with a capacitor/inductor.

Figure 9a presents the principle of the gyrator circuit while Figure 9b presents its small-signal model. Each transistor in this simplified view is actually an Operational Transconductance Amplifier (OTA). We note that it is highly recommended to use one-stage OTAs as multiplestages OTAs introduce more nodes thus leading to greater risks of instability (for possible topologies of one-stage OTA circuits see Appendix B). In contrast to positive active inductors, which use two OTAs with $g_{m}$ of different signs, negative inductors use two OTA with $g_{m}$ of the same sign.

Considering a simplified model, without parasitic capacitors, the input impedance is given by:

$$
Z_{\mathrm{gyr}}=\frac{r_{0}\left(r_{0}+Z_{L}\right)}{r_{0}-Z_{L}\left(g_{m}^{2} r_{0}^{2}-1\right)} \approx \frac{1}{g_{m}^{2}}\left(-\frac{1}{r_{0}}-\frac{1}{Z_{L}}\right) .
$$

The final approximation was obtained by assuming both $g_{m}^{2} r_{0}^{2} \gg 1$ and $\left|g_{m}^{2} r_{0} Z_{L}\right| \gg 1$. Thus, for the gyrator, both reactive and resistive parts of the impedance are negative and decrease with $g_{m}^{2}$.

At first glance, it would then seem as though one only need consider a pure capacitance $\left(Z_{L}=1 / j C \omega\right)$ together with a large $g_{m}$. However, when one goes beyond the simplified model, resistive and reactive parts can no longer be dissociated. In the following, we consider the full model, i.e., including parasitic capacitors and without any approximation (for the complete expression of the input impedance see Appendix A.2).

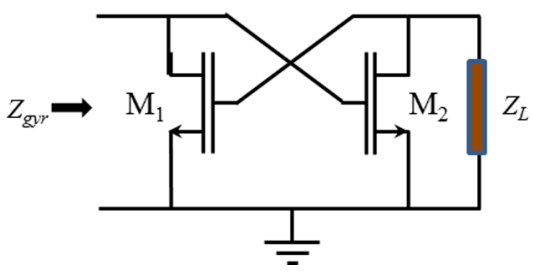

a)

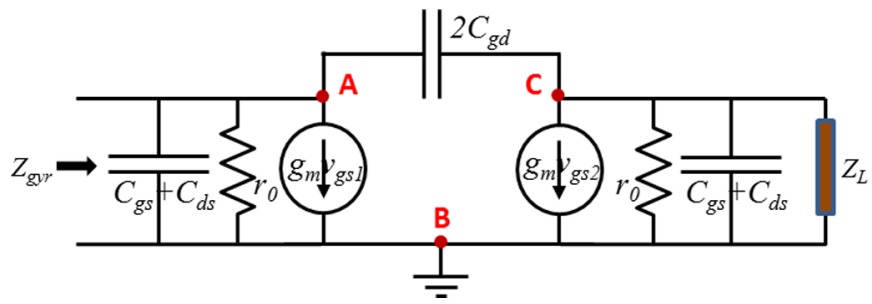

b)

Fig. 9. a) Gyrator circuit with MOSFET transistors. b) Smallsignal model of the gyrator circuit.

In the gyrator circuit, three parameters are readily controllable: $r_{0}, g_{m}$ and $C$ via varactors $\left(r_{0}\right.$ has been kept constant at $2 \mathrm{k} \Omega$ for simplicity). Even within the full model, they afford enough degrees of freedom to cancel the real part of the NF impedance at any frequency of interest. In contrast to the Linvill though, a thorough knowledge of parasitic capacitances is required to achieve such cancellation. To illustrate this fact, we consider a specific example. Figure 10 presents results that were obtained for three different combinations of $g_{m}$ and $C$. All combinations ensure that the negative inductance equals $-32.2 \mathrm{nH}$ at $250 \mathrm{MHz}$ but the real part cancels at different frequencies $\left(f_{\text {Re } 0}\right)$. We see that optimal values of $g_{m}$ and $C$ do not follow any simple law.

Conclusions of this section are threefold. First, a negative inductance can be obtained by introducing a capacitor in the load impedance. Second, cancellation of the real part can be achieved by a suitable combination of $g_{m}$ and $C$. Finally, the real part is overall much smaller than for the Linvill and, thus, the exact frequency at which it does cancel is not as critical as its minimization to achieve a large bandwidth.

\subsection{Linvill vs. Gyrator}

Both topologies can lead to the desired values of negative inductance, but do not present the same advantages.

Concerning the quality factor $Q=\operatorname{Im}\left(Z_{\mathrm{NF}}\right) / \operatorname{Re}\left(Z_{\mathrm{NF}}\right)$, Linvill circuits presents a real part that is relatively large compared to gyrators, hence a much lower quality factor. However, it is easier to cancel the real part around the frequency of interest with Linvills than with gyrators, thanks to the compensation resistor $R$. Still, it does not actually affect performances of gyrator circuits as they do not present a steep variation of the real part with frequency. Concerning the chip area, Linvill circuits are clearly worse than gyrators as they require an inductance. Gyrator circuits are more compact and, additionally, offer the possibility to tune $g_{m}$ via the bias current to change the value of the negative inductance. Concerning stability, it 

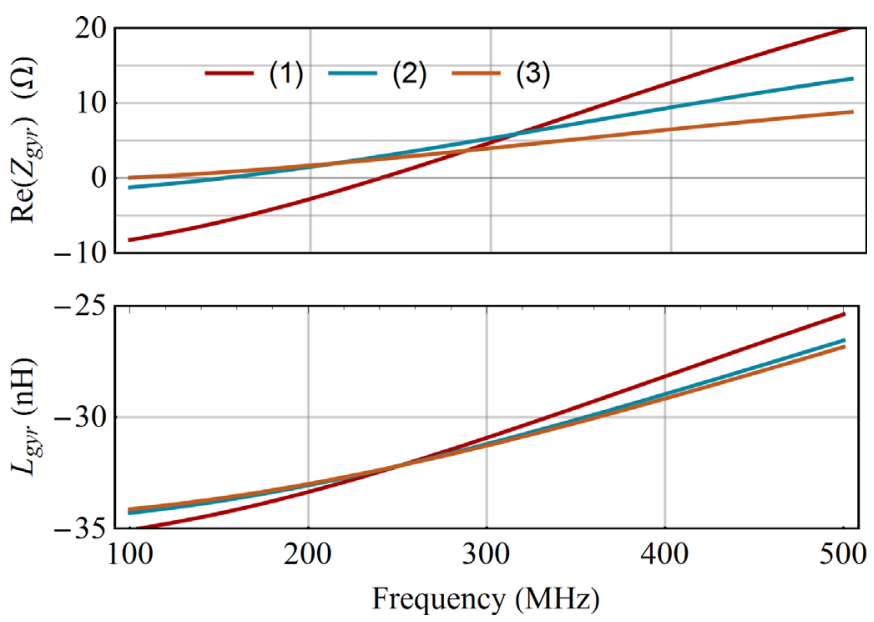

Fig. 10. Input impedance of the gyrator circuit, $Z_{\mathrm{gyr}}=R e$ $\left(Z_{\text {gyr }}\right)+j L_{\text {gyr }} \omega(1) g_{\mathrm{m}}=7 \mathrm{mS}, C=0.87 \mathrm{pF}, f_{\mathrm{Re} 0}=239 \mathrm{MHz} \quad(2)$ $g_{\mathrm{m}}=15 \mathrm{mS}, \quad C=7.0 \mathrm{pF}, \quad f_{\operatorname{Re} 0}=152 \mathrm{MHz} \quad(3) \quad g_{\mathrm{m}}=30 \mathrm{mS}$, $C=30 \mathrm{pF}, f_{\operatorname{Re} 0}=96 \mathrm{MHz}$.

will be shown in what follows that the choice of configuration (in shunt or in series) is a key point for the stability of the whole metamaterial.

\section{Stability}

Stability of non-Foster circuits is a recurrent topic in the literature [48-50]. The approach used in this paper to systematically detect the emergence of instability in a circuit is similar to that proposed in [51]. In order for this paper to be self-contained, we summarize its main steps below.

In general, a linear system can be considered stable if a Bounded Input results in a Bounded Output (BIBO). Control theory states that it is possible to determine the $\mathrm{BIBO}$ nature of a closed-loop system by studying poles of its transfer function: All poles must have a negative real part, i.e., belong to the Left Half Plane (LHP).

For a linear circuit, stability occurs if no current or voltage output diverges or oscillates for any applied input. This must hold true at all nodes. A unitary impulsion $V(s)$ or $I(s)$ must thus be applied to each node, making sure that the circuit returns to its initial state once the impulsion has died out. The choice is made as follows [51]:

- If the node of the circuit is connected to a virtual ground then a voltage impulsion $V(s)$ is introduced in the circuit. Since $I(s)=Y(s)$, the current $i(t)$ will diverge if poles of $\mathrm{Y}$ $(\mathrm{s})$, zeros of $\mathrm{Z}(\mathrm{s})$, have positive real parts. Thus, it is an admittance analysis;

- If the node of the circuit is not connected to a virtual ground then a current impulsion $I(s)$ is introduced in the circuit. Since $V(s)=Z(s)$, the voltage $v(t)$ will diverge if poles of $\mathrm{Z}(\mathrm{s})$, zeros of $\mathrm{Y}(\mathrm{s})$, have positive real parts. It is an impedance analysis.

In our case, we have observed that no exact pole-zero cancellation occurs for either Linvill or gyrator circuits and, thus, all nodes have the same poles. Consequently, stability need not be studied at every node and any given node can be chosen [51].
In the following we proceed to a two-step stability study of Linvill and gyrator circuits. First, we consider their intrinsic stability, i.e., the stability of the NF circuit alone. Second, we consider the stability of Linvill circuits connected to AMMs and gyrator circuits connected to AMCs, respectively.

\subsection{Intrinsic stability of Linvill circuits}

The structure of the Linvill circuit is very close to that of a cross-coupled oscillator. Therefore, stability is critical.

All nodes being equivalent, we choose to consider stability at node A (Fig. 7b). Since this node is connected to a virtual ground, a voltage impulsion is applied and we proceed to an admittance analysis. Within the simplified model, the only pole of $Y_{\text {Linv }}$ is given by:

$$
s_{0}=\frac{2 r_{0}-R\left(g_{m} r_{0}-1\right)}{L\left(g_{m} r_{0}-1\right)} \text {. }
$$

From equation (7), conditions for intrinsic stability can be deduced. Hereon, we assume that the product $g_{m} r_{0}$ is large enough to achieve a negative inductance $\left(g_{m} r_{0}>1\right)$.

When $R$ tends towards zero, the real part of this pole is positive (unstable). When $R$ increases, the real part of this pole becomes negative (stable), as soon as $R>2 r_{0}$ / $\left(g_{m} r_{0}-1\right)$. With the values from Figure 8, stability is achieved for compensation resistors larger than $68 \Omega$ (this condition no longer strictly holds within the full model). In short, Linvill circuits often present an intrinsic instability in the typical negative inductance configuration.

\subsection{Stability of Linvill circuits coupled to AMMs}

The intrinsic instability of the Linvill circuit can be compensated by connecting it to a suitable impedance. Because instability is due to the pole of the Linvill admittance, stability can be obtained by introducing in series a positive inductance greater than the absolute value of the Linvill's negative inductance (total inductance $\left.L_{t}>0\right)$. Hence, Linvill circuits are compatible with AMMs but not with AMCs.

Stability is thus analyzed when a Linvill circuit is coupled to a SRR array (Fig. 1). The electrical model of the $\mathrm{SRR}$ is composed of a transformer, $L_{p}=45 \mathrm{nH}, L_{1}=$ $31.5 \mathrm{nH}, M=18.5 \mathrm{nH}$, in series with a resistance $R_{p}=3 \Omega$ and a capacitor $C_{p}=18 \mathrm{pF}$, leading to a resonance at $530 \mathrm{MHz}$. An equivalent resistance $R_{r}=377 \Omega$ models the characteristic impedance of air. The equivalent circuit considered for the stability study corresponds to Figure 1 with $Z_{\mathrm{NF}}$ replaced by the circuit on Figure $7 \mathrm{~b}$.

The complete system, as well as the possible nodes of study, is presented in Figure 11. If the stability study is undertaken at nodes B, C or D, an impedance analysis (current impulsion) will have to be carried out whereas, if it is undertaken at node $\mathrm{A}$, it will have to be an admittance analysis (voltage impulsion).

Here, node B has been chosen for analysis. The analysis of poles loci is carried out only with respect to $L$, as $g_{m}$ is set to its maximum value $(22 \mathrm{mS})$ and $R$ is chosen to cancel the real part of $Z_{\text {Linv }}$ at $530 \mathrm{MHz}$ (adjusted for each value of $L$, it varies from 90 to $60 \Omega$ ). 


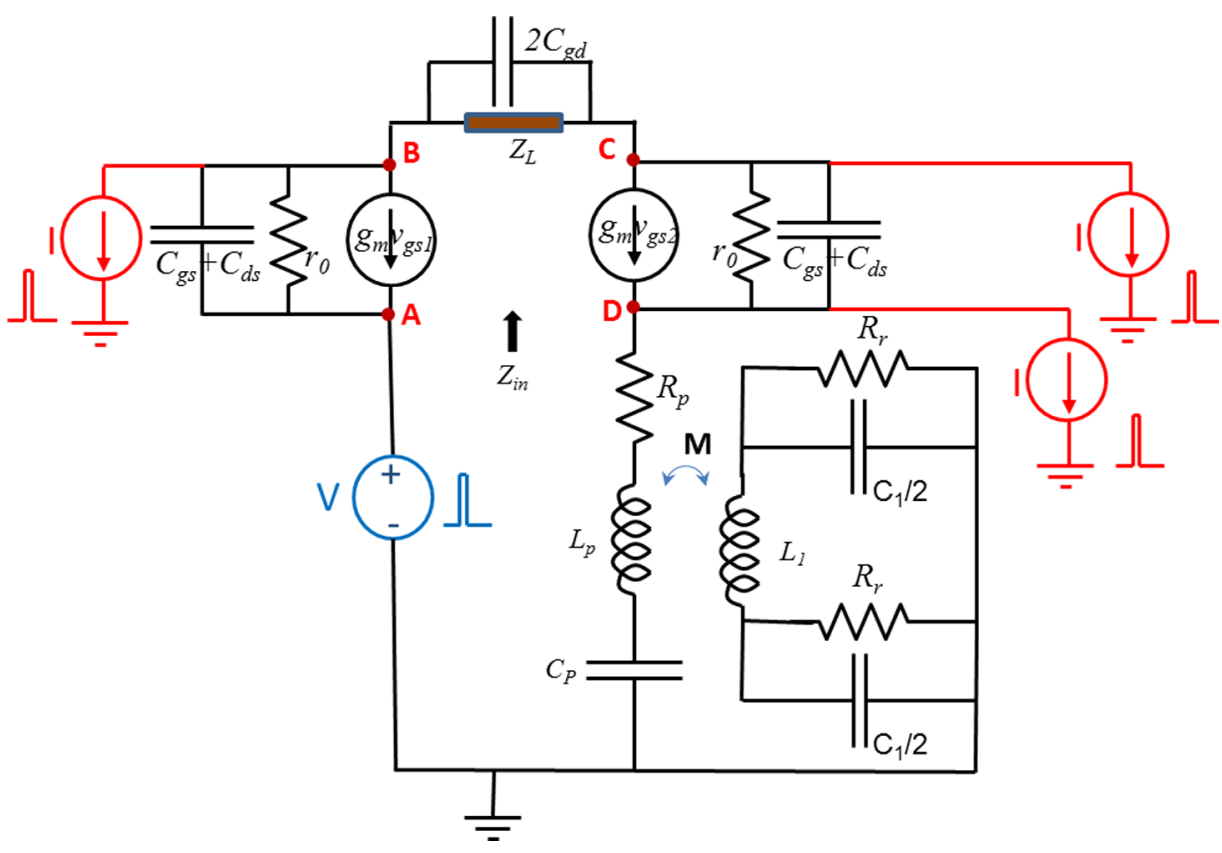

Fig. 11. Equivalent circuit of the Linvill NIC coupled to the SRR array (AMM). All four nodes and their respective impedance/ admittance analysis are represented.

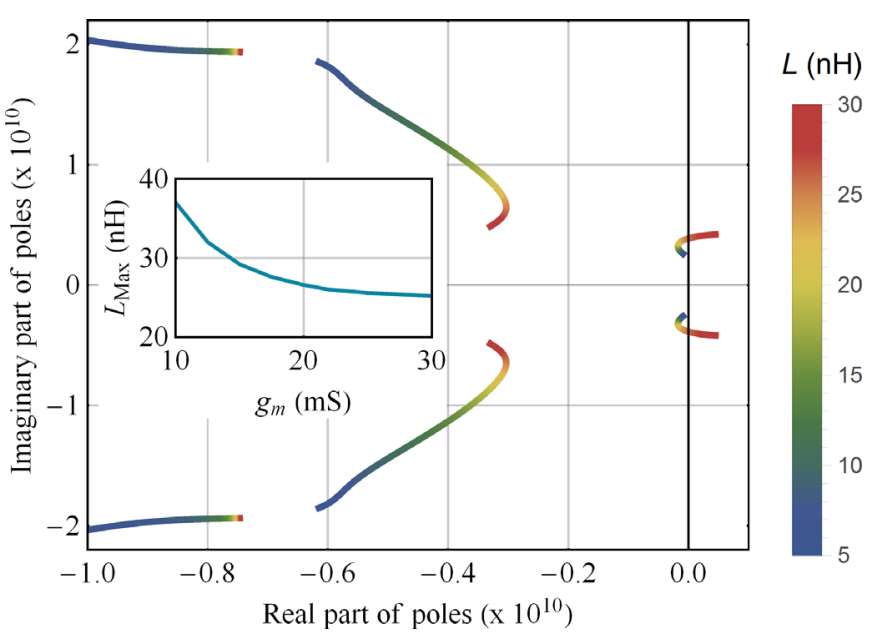

Fig. 12. Poles loci at node B. $g_{\mathrm{m}}$ is constant $(22 \mathrm{mS})$. To cancel the real part of $Z_{\text {Linv }}$ at $530 \mathrm{MHz}, R$ varies from 90 to $60 \Omega$ when $L$ goes from 5 to $30 \mathrm{nH}$. The inset shows the evolution of $L_{\max }$ with $g_{\mathrm{m}}$.

Figure 12 shows poles loci obtained for $L$ varying between 5 and $30 \mathrm{nH}$, leading to a negative inductance varying from -2 to $-30 \mathrm{nH}$. It can be seen that a value of $L$ greater than $L_{\max }=26 \mathrm{nH}$, which corresponds to $L_{\mathrm{Linv}}=$ $-28.2 \mathrm{nH}$ at $530 \mathrm{MHz}$, places two poles in the Right Half Plane (RHP). Since a total inductance close to zero $\left(L_{p}=45 \mathrm{nH}\right)$ is critical to get a significant bandwidth improvement, subpar performances can be anticipated. The inset presents the evolution of $L_{\max }$ with $g_{m}$ : increasing $g_{m}$ further leads to a slowly decreasing $L_{\max }$.

There is an inherent difficulty in coupling Linvill circuits to AMMs: to obtain small resistances a large $g_{m}$ is needed but with a large $g_{m}$ the possible negative inductances are limited by stability considerations.

\subsection{Intrinsic stability of gyrator circuits}

All nodes being equivalent, we choose to consider stability at node A (Fig. 9b). Since this node is not connected to a virtual ground, a current impulsion is applied and we proceed to an impedance analysis. Within the simplified model, the only pole of $Z_{\mathrm{gyr}}$ is given by:

$$
s_{0}=\frac{g_{m}^{2} r_{0}^{2}-1}{r_{0} C} .
$$

From equation (8), conditions for intrinsic stability can be straightforwardly deduced. When $g_{m}>1 / r_{0}$, the real part of this pole is positive (unstable) and the impedance is mostly inductive. When $g_{m}<1 / r_{0}$, the real part of this pole is negative (stable) and the impedance is mostly resistive.

It means that as long as the impedance is mostly inductive the negative inductance created by the gyrator circuit is intrinsically instable.

\subsection{Stability of gyrator circuits coupled to AMCs}

The intrinsic instability of the gyrator circuit can be compensated by connecting it to a suitable impedance.

Because instability is due to the pole of the gyrator impedance, stability can never be restored by connecting a positive inductor in series [50]. However, a stable configuration can be obtained in shunt with a positive inductance smaller than the absolute value of the gyrator's negative inductance (total inductance $L_{t}>0$ )[50]. Hence, gyrator circuits are compatible with AMCs but not with AMMs.

Stability is thus analyzed when a gyrator circuit is coupled to a patch array (Fig. 4). The electrical model of the patch array is composed of a capacitor $\left(C_{\mathrm{g} 0}=0.25 \mathrm{pF}\right)$ 


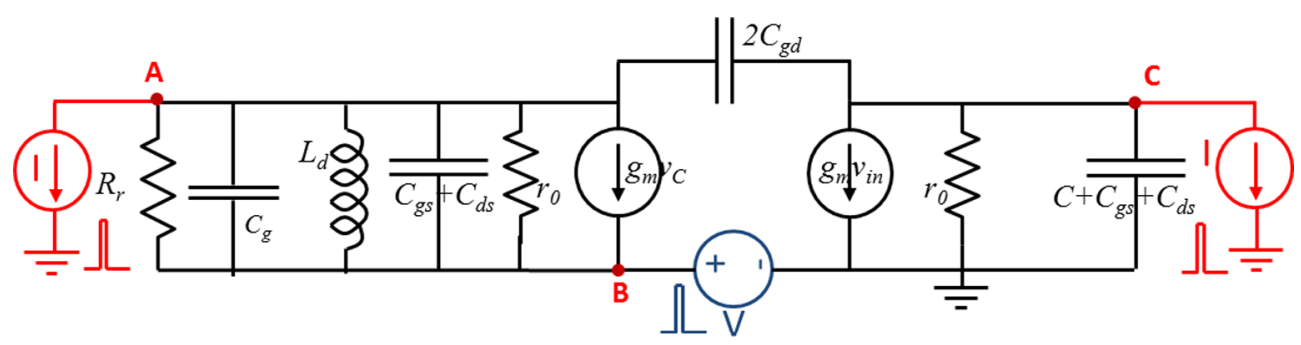

Fig. 13. Equivalent circuit of the gyrator NII coupled to the patch array (AMC). All three nodes and their respective impedance/ admittance analysis are represented.

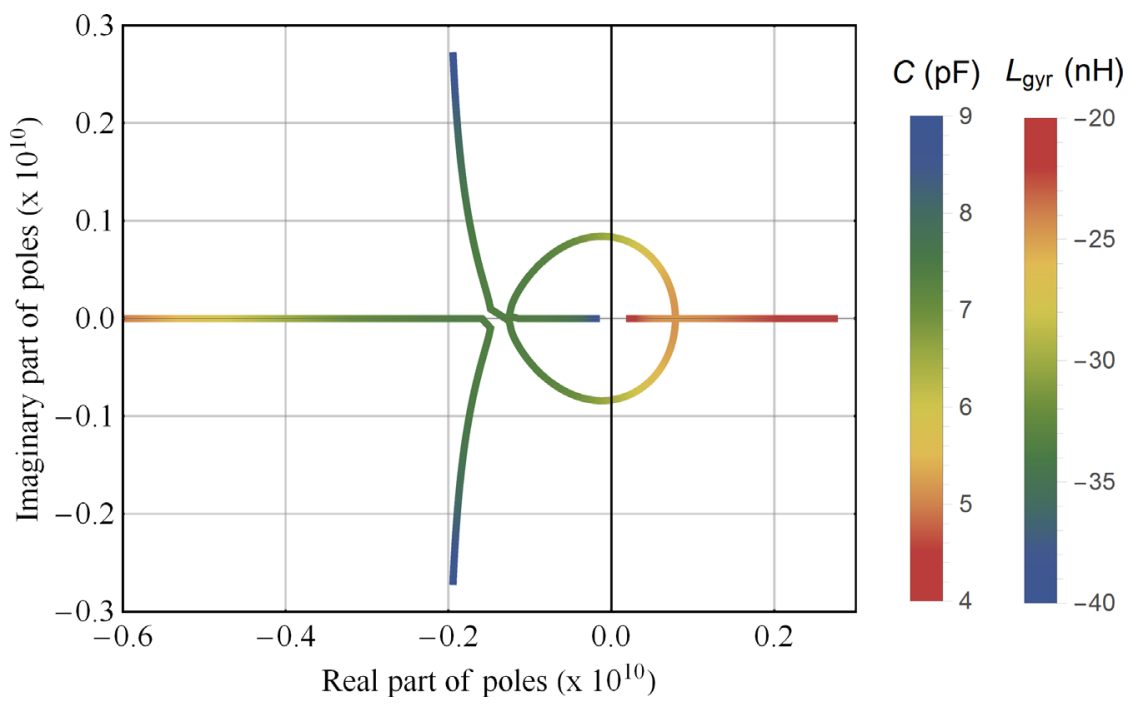

Fig. 14. Poles loci at node $\mathrm{A}\left(g_{\mathrm{m}}=15 \mathrm{mS}, C\right.$ varies from $9 \mathrm{pF}$ to $4 \mathrm{pF}$ when $L_{\text {gyr }}$ goes from $-40 \mathrm{nH}$ to $\left.-20 \mathrm{nH}\right)$.

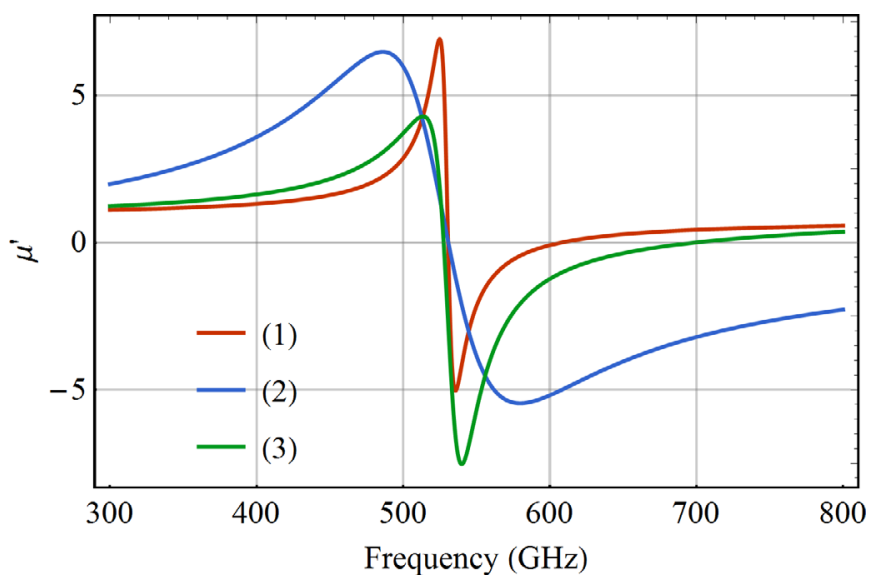

Fig. 15. Effective permeability of a SRR array vs. frequency for the following three configurations: (1) Initial SRR array, $C_{p}=2 \mathrm{pF} \quad(2)$ Ideal negative inductance, $C_{p}=18 \mathrm{pF}, L_{\text {neg }}=$ $-40 \mathrm{nH}, R_{\mathrm{NF}}=0 \Omega(3)$ Stable Linvill circuit $C_{p}=4,4 \mathrm{pF}, L_{\mathrm{neg}}=$ $-24.4 \mathrm{nH}, R_{\mathrm{NF}}=66 \Omega$.

in shunt with an inductor $L_{d}=31.4 \mathrm{nH}$. An equivalent resistance $R_{r}=377 \Omega$ models the impedance of air. The equivalent circuit considered for the stability study corresponds to Figure 1 with $Z_{\mathrm{NF}}$ replaced by the circuit on Figure 9b.

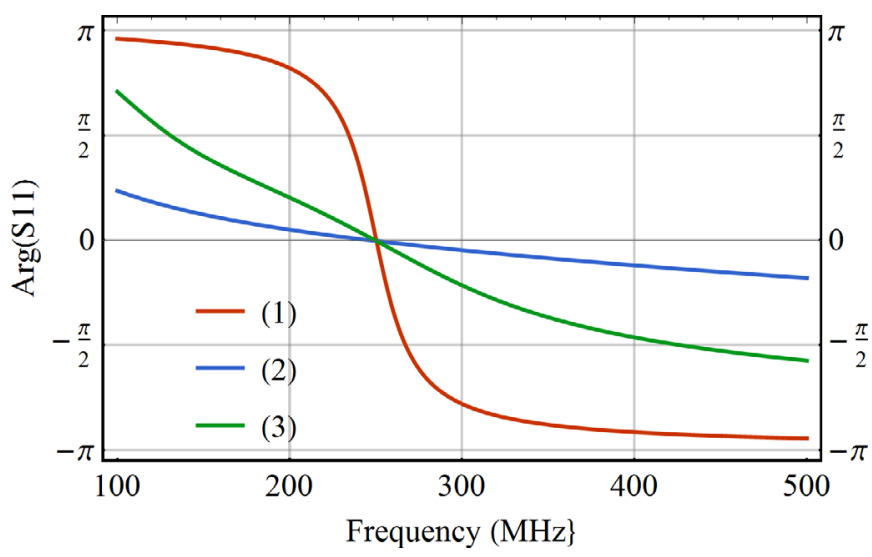

Fig. 16. Phase on reflection of a patch array vs. frequency for the following three configurations: (1) Initial patch array, $C_{g}=C_{\mathrm{gl}}=$ $12.8 \mathrm{pF}$ (2) Ideal negative inductance, $C_{g}=C_{\mathrm{g} 0}=0.25 \mathrm{pF}, L_{\mathrm{neg}}=$ $-32.2 \mathrm{nH}, R_{\mathrm{NF}}=0 \Omega$ (3) Stable gyrator circuit, $C_{\mathrm{g} 0}=0.25 \mathrm{pF}$, $L_{\text {neg }}=-31.9 \mathrm{nH}, R_{\mathrm{NF}}=3.2 \Omega$.

The complete system, as well as the possible nodes of study, is presented in Figure 11. If the stability study is undertaken at nodes $\mathrm{A}$ or $\mathrm{C}$, an impedance analysis (current impulsion) will have to be carried out whereas, if it is undertaken at node $\mathrm{B}$, it will have to be an admittance analysis (voltage impulsion). 
Here, node A has been chosen for analysis. The analysis of poles loci is carried out with respect to both $g_{m}$ and $C$.

Figure 12 shows poles loci for $g_{m}=15 \mathrm{mS}$ and $C$ varying from 9 to $4 \mathrm{pF}$, leading to a negative inductance varying from -40 to $-20 \mathrm{nH}$.

All values of $L_{\text {gyr }}$ such as $\left|L_{g y r}\right|>L_{d}$ lead to a stable configuration. Nevertheless, the limit value occurring for $C_{\min }=6.3 \mathrm{pF}$, which corresponds to $L_{\mathrm{gyr}}=-29.7 \mathrm{nH}$, exceeds somewhat this condition. Two reasons explain this behavior:

- The impedance of the air appears in shunt with $r_{0}$ and decreases the quality factor of the system gyrator/AMC.

- The real part of the gyrator increases with the frequency (cf. Figure 10).

Indeed, it's possible to generate a stable inductance close to the short-circuit inductance $L_{d}$ which is critical to get a significant bandwidth improvement. Variations of $g_{m}$ or of $r_{0}$ do not modify this observation.

In contrast with the result obtained for the Linvill/ AMM configuration, there is no intrinsic difficulty in coupling gyrator circuits to AMCs: small resistances are intrinsic while stability does not preclude from obtaining the required inductances.

\section{Performances of metamaterials coupled with non-Foster circuits}

In the following, stable Linvill and gyrator circuits are coupled with SSR and patch arrays, respectively, to evaluate the bandwidth enhancement that can be attained.

\subsection{AMMs coupled with Linvill circuits}

Parameters of the considered Linvill circuit are: $g_{m}=$ $22 \mathrm{mS}, R=66 \Omega$, and $L=25 \mathrm{nH}$. In this case, a negative inductance of $-24.4 \mathrm{nH}$ is generated. The corresponding effective permeability is compared to that obtained with an ideal negative inductance and a stable Linvill circuit (Fig. 15). Note that $C_{p}$ was chosen equal to $4.4 \mathrm{pF}$ in order to keep the resonance frequency at $530 \mathrm{MHz}$.

It appears that, even with an optimized Linvill circuit, only a modest bandwidth enhancement can be achieved. Furthermore, there are no simple solutions in sight to improve this result. Decreasing parasitic capacitances, e.g. using a different transistor technology, can lead to the migration of other poles to the RHP and, thus, instability.

\subsection{AMC coupled with gyrator circuits}

Parameters of the considered gyrator circuit are: $g_{m}=$ $15 \mathrm{mS}$ and $C=7,3 \mathrm{pF}$. In this case, a negative inductance of $-31.9 \mathrm{nH}$ is generated. The corresponding phase on reflection is compared to that obtained with an ideal negative inductance and a stable gyrator circuit (Fig. 16).

To quantify the performance of this non-Foster AMC, we consider the slope as the figure of merit (see Eq. (4)). An ideal inductor would decrease this slope by a factor of 51 . A gyrator-based negative inductor does decrease this slope by a factor 5.1. Although far from optimal, this gain is still very interesting because it is the performance that would be achieved with a five times thicker AMC.

\section{Conclusions}

In this paper, we investigated all requirements imposed on non-Foster circuits by bandwidth enhancement. First, we considered two metamaterial families, namely AMMs and AMCs. The investigation of both metamaterial families showed that the achievement of non-Foster metamaterials requires negative inductances, to compensate for intrinsic positive inductances, and extremely small resistances, to avoid attenuation or amplification. Then, we investigated two practical electronic architectures: Linvill and gyrator circuits. Stability considerations lead us to connect Linvill circuits to AMMs and gyrator circuits to AMCs. It was shown that the input impedance of Linvill circuits is dictated solely by the load impedance whereas, for gyrator circuits, both load impedance and transconductance play a role.

Conclusions are different for the two types of circuits. For Linvill circuits, stability is achieved only far away from the optimum. This represents a major limitation and, thus, this type of NF circuit does not bring about any gain in performance for the considered application. For gyrator circuits, no such issues were met. Yet, achieving both small resistances and large/negative inductances simultaneously and on a large bandwidth proved challenging. In the end, the performance of this type of NF circuit was still more than five times better than that of conventional metamaterials.

\subsection{Implications and influences}

In the last ten years, several authors have suggested coupling metamaterials with non-Foster circuits to increase the operational frequency bandwidth. However, up to now, the number of convincing demonstrations based on non-Foster metamaterials has been very limited. To go beyond these first attempts and get a deeper understanding of the difficulty in coupling metamaterials with non-Foster circuits, we combined the expertise of scientists working in the fields of metamaterials and integrated circuits.

On two representative examples, this paper suggests a methodology to study the coupling of metamaterials and non-Foster circuits taking into account the fundamental issue of the stability. These examples show the utmost importance of selecting the appropriate electronic architecture for a given metamaterial. Besides, they also show the compelling need to consider detailed equivalent circuits of these architectures, even down to parasitic terms.

We firmly believe that this paper, by bringing together complementary expertise, will be very valuable for all scientists striving to combine metamaterials with electronics circuits.

\section{Appendix A}

The purpose of this appendix is to provide the full equations, in all of their glorious complexity, for the Linvill and gyrators circuits. 

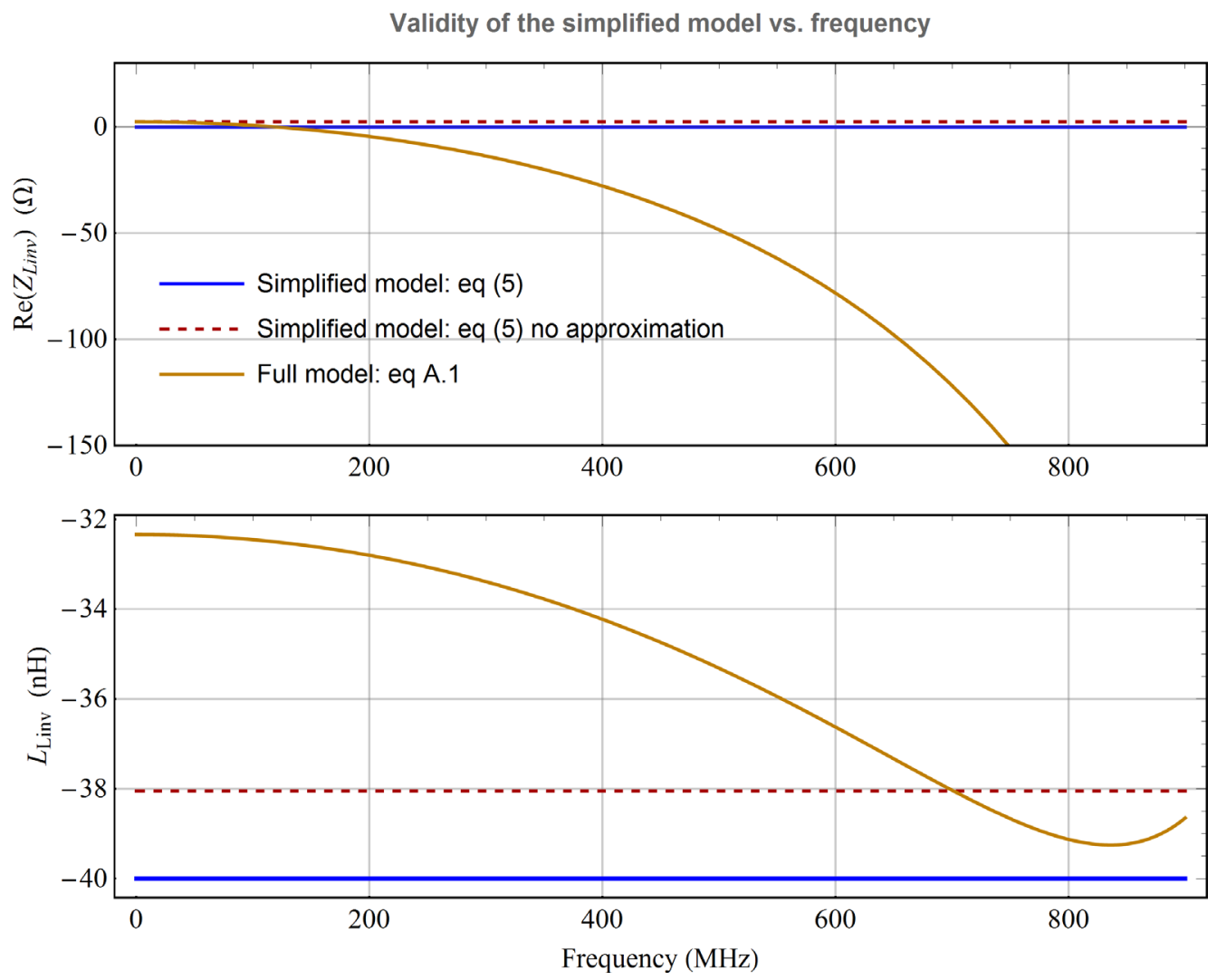

Fig. A.1. Comparison of the simplified and full model of the Linvill circuit from 100 to $900 \mathrm{MHz}$. Circuit parameters are $g_{\mathrm{m}}=20 \mathrm{mS}$, $R=100 \Omega$, and $L=40 \mathrm{nH}$. (Top) Real part of the input impedance. (Bottom) Equivalent inductance of the input impedance.

\section{A.1 Linvill}

The input impedance of the full model, with compensation resistor, parasitic capacitors and no approximation is given by:

See Eq. (A.1) at the bottom

Simplified and full models are compared on Figure A.1 for a specific case $\left(g_{m}=20 \mathrm{mS}, R=100 \Omega, L=40 \mathrm{nH}\right.$, same parameters as Figure 8). All other parameters are given in Section 3.

As can be seen from Figure A.1, there is little to no impact of the approximation on the simplified model except for a small upwards shift of the inductance. However, only the full model should be used at frequencies above $500 \mathrm{MHz}$ since resistive parts of the input impedance for simplified and full models strongly deviate from each other afterwards.

\section{A.2 Gyrator}

The input impedance of the full model, with compensation resistor, parasitic capacitors and no approximation is given by:

See Eq. (A.2.1) at the bottom

Simplified and full models are compared on Figure A.2 for a specific case $\left(g_{m}=15 \mathrm{mS}, C=7 \mathrm{pF}\right.$, same parameters as Figure 10). All other parameters are given in Section 3.

As can be seen from Figure A.2, there is a small but significant impact of the approximation on the simplified model as it predicts only positive resistance, thereby missing potential instabilities. However, unlike the Linvill, the simplified model of the gyrator is valid on a very wide frequency range since the overall deviation is much smaller.

$$
\begin{aligned}
& Z_{\text {Linv }}=\frac{2 r_{0}+R\left(1 g_{m} r_{0}\right)+j \omega R r_{0}\left(4 C_{\mathrm{gd}}+C_{\mathrm{par}}\right) j \omega L\left(g_{m} r_{0} 1\right) L r_{0}\left(C_{\mathrm{par}}+4 C_{\mathrm{gd}}\right) \omega^{2}}{\left(g_{m} r_{0}+1+j \omega C_{\mathrm{par}} r_{0}\right)\left(1+j 2 R C_{\mathrm{gd}} \omega 2 L C_{\mathrm{gd}} \omega^{2}\right)} . \\
& Z_{\mathrm{gyr}}=\frac{r_{0}\left(1+j \omega r_{0}\left(4 C_{\mathrm{gd}}+C_{L}\right)\right.}{1 g_{m}^{2} r_{o}^{2}+j \omega r_{0}\left(4 C_{\mathrm{gd}}\left(1+g_{m} r_{0}\right)+C_{L}+C_{p a r}\right) \omega^{2} r_{o}^{2}\left(2 C_{\mathrm{gd}}\left(C_{L}+C_{\mathrm{par}}\right)+C_{L} C_{\mathrm{par}}\right)} . \\
& \text { with } C_{L}={ }_{\text {par }}+{ }^{2}
\end{aligned}
$$



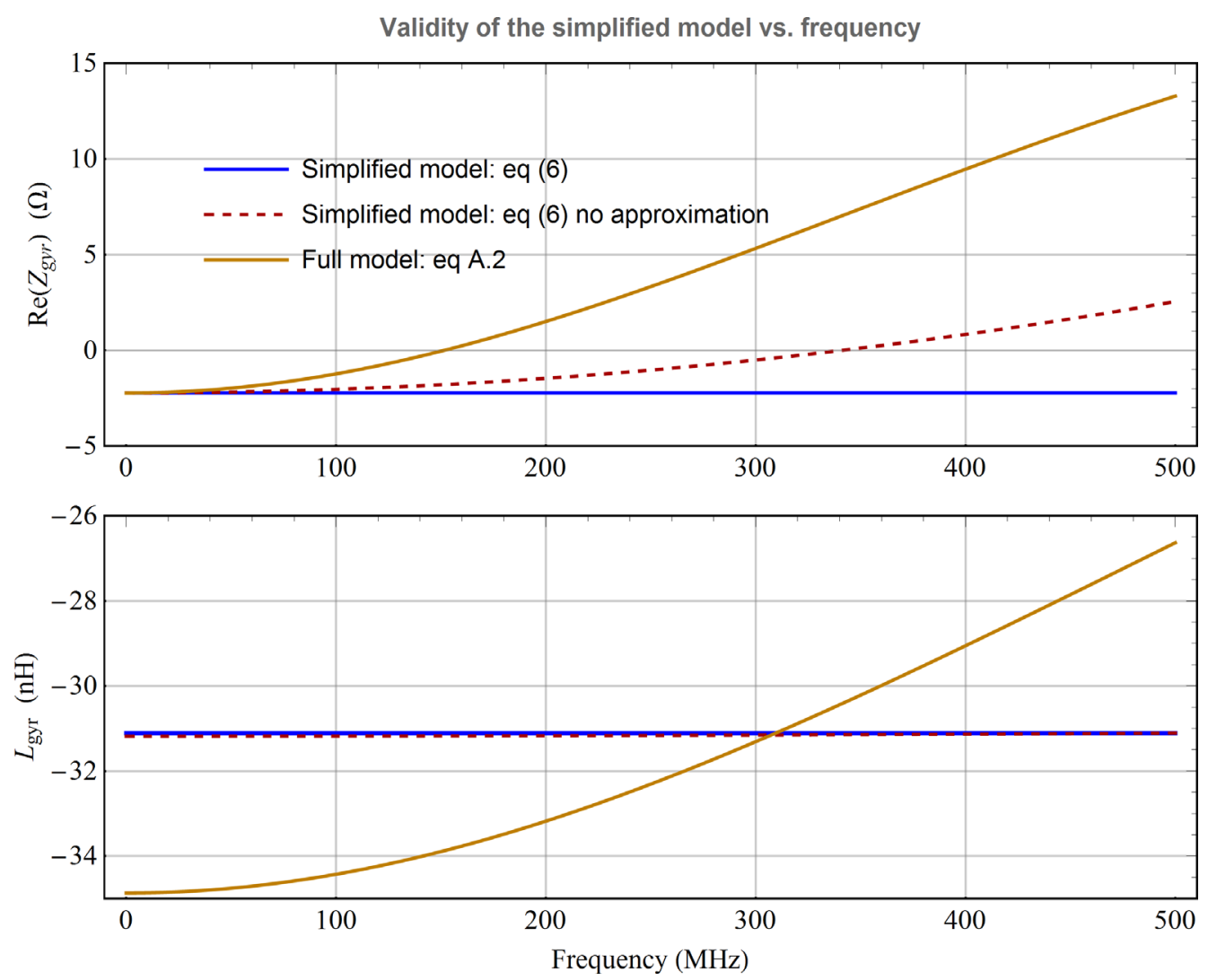

Fig. A.2. Comparison of the simplified and full model of the gyrator circuit from 100 to $500 \mathrm{MHz}$. Circuit parameters are $g_{\mathrm{m}}=15 \mathrm{mS}$, and $C=7 \mathrm{pF}$. (Top) Real part of the input impedance. (Bottom) Equivalent inductance of the input impedance.
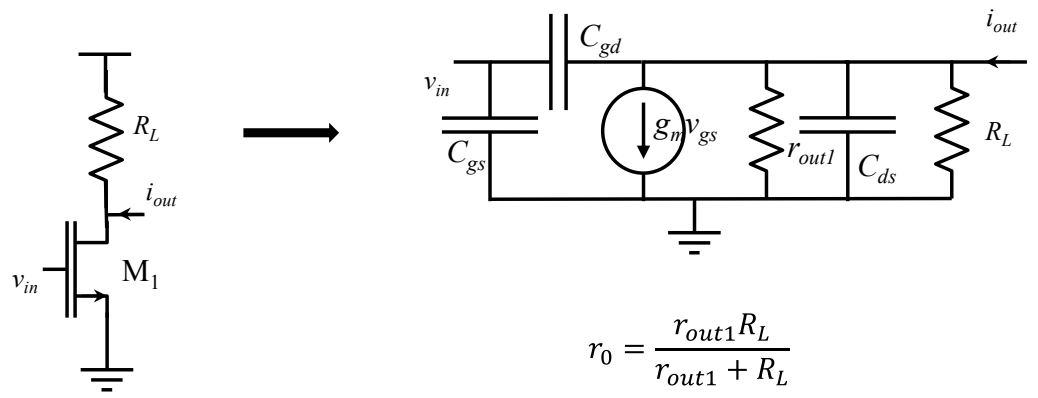

$$
r_{0}=\frac{r_{\text {out } 1} R_{L}}{r_{\text {out } 1}+R_{L}}
$$

a)
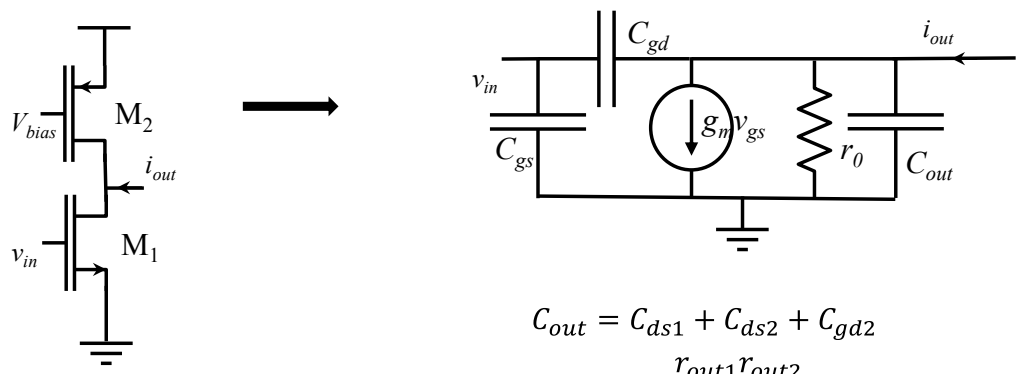

$$
\begin{gathered}
C_{\text {out }}=C_{d s 1}+C_{d s 2}+C_{g d 2} \\
r_{0}=\frac{r_{\text {out } 1} r_{\text {out } 2}}{r_{\text {out } 1}\left(1+g_{m 2} r_{\text {out } 2}\right)+r_{\text {out } 2}}
\end{gathered}
$$

b)

Fig. B.1. Single Input Single Output (SISO) configurations of OTA. 


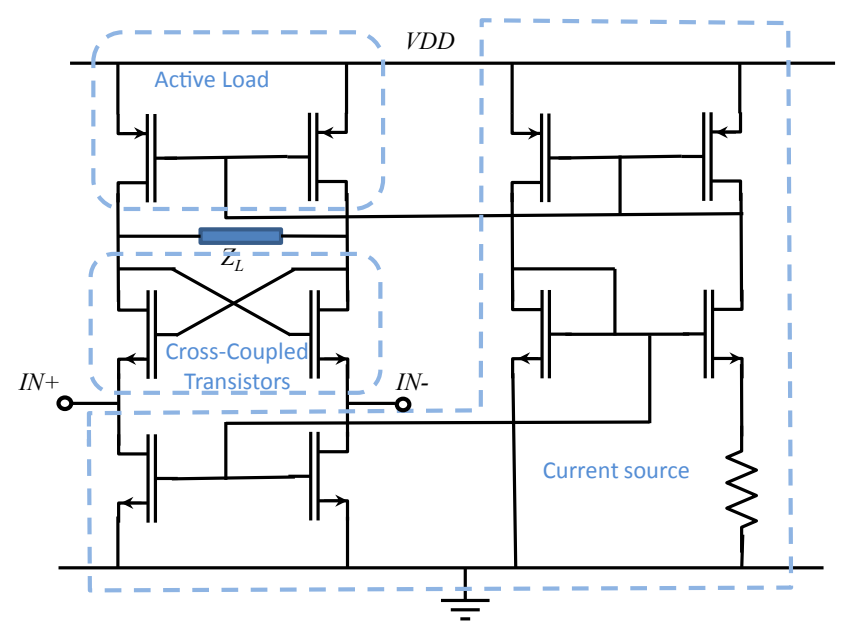

a)

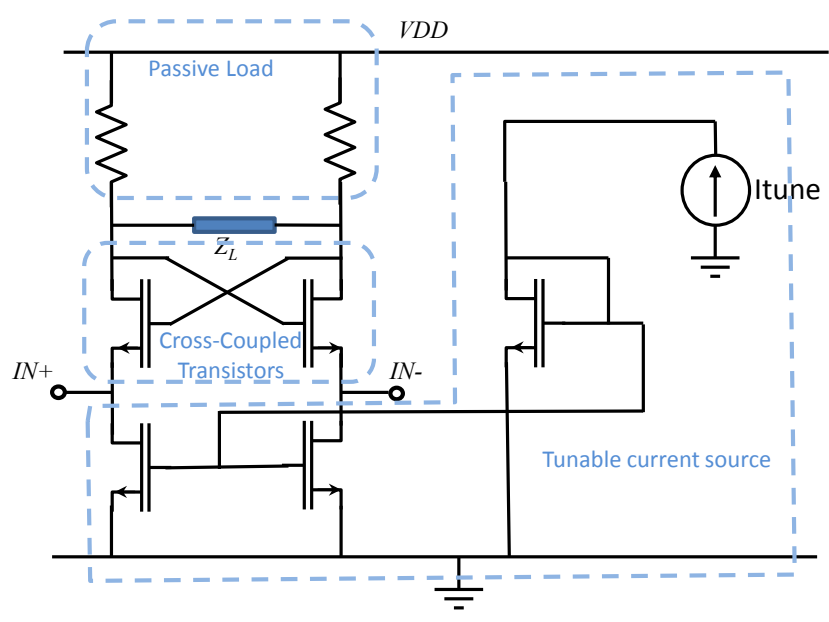

b)

Fig. B.2. Linvill circuit: a) published in [42], b) published in [39].

\section{Appendix B}

The purpose of this appendix is to provide to the reader a synthetic overview on possible circuits for the gyrator and the Linvill topologies. It could not be an exhaustive presentation.

\section{B.1 Gyrator}

The gyrator topology is based on two OTA (Operational Transconductance Amplifier). In the case of Non Foster, due to the high risk of instability it is better to limit the number of nodes and then the number of stages in the OTA. Two basic configurations are presented in Figure B.1. The equivalent small signal model is coherent with the small signal model of a MOSFET transistor. It means that the studies presented in this article are valid for these configurations, by only modifying the values of parameters of the small signal model, particularly $r_{0}$. An example of gyrator based on two one stage inverter based OTA is proposed in [52]

\section{B.2 Linvill}

The Linvill circuit has been achieved many times with varying technologies (bipolar transistors or MOSFET, integrated or with lumped elements). We propose in Figure B.2 two MOSFET-based recently published circuits $[42,39]$. As it can be seen on the figure, the drains of the cross-coupled transistors could be connected to transistors or resistors. So in the small signal model of the MOSFET it will change the order of magnitude of $r_{0}$.

\section{References}

1. D. Youla, L. Castriota, H. Carlin, IRE Trans. Circ. Theor. 6, $102(1959)$

2. S.A. Tretyakov, Microw. Opt. Technol. Lett. 31, 163 (2001)
3. S.A. Tretyakov, S.I. Maslovski, IEEE Antennas Propag. Mag. 49, 37 (2007)

4. S. Hrabar et al., Appl. Phys. Lett. 99, 254103 (2011)

5. S. Hrabar, I. Krois, A. Kiricenko, Metamaterials 4, 89 (2010)

6. P.-Y. Chen, C. Argyropoulos, A. Alù, Phys. Rev. Lett. 111, 233001 (2013)

7. D.F. Sievenpiper, IEEE Antennas Wirel. Propag. Lett. 10, 231 (2011)

8. S. Saadat et al., IEEE Trans. Antennas Propag. 61, 1210 (2013)

9. F. Gao et al., Electron. Lett. 50, 1616 (2014)

10. Y. Ding, V. Fusco, Microw. Opt. Technol. Lett. 54, 2111 (2012)

11. M. Barbuto et al., IEEE Trans. Antennas Propag. 61, 1219 (2013)

12. C.G. Montgomery, R.H. Dicke, E.M. Purcell, Principles of microwave circuits (Iet, 1948)

13. A.D. Harris, G.A. Myers, DTIC Document, 1968

14. J.T. Aberle, R. Loepsinger-Romak, Synth. Lect. Antennas 2, 1 (2007)

15. S.E. Sussman-Fort, R.M. Rudish, IEEE Trans. Antennas Propag. 57, 2230 (2009)

16. C.R. White, J.S. Colburn, R.G. Nagele, IEEE Antennas Wirel. Propag. Lett. 11, 584 (2012)

17. O.O. Tade, P. Gardner, P.S. Hall, in: 42nd European Microwave Conference (EuMC), 2012, p. 613

18. S.D. Stearns, in: IEEE International Symposium on Antennas and Propagation (APSURSI), 2011, p. 1942

19. M.M. Jacob, J. Long, D.F. Sievenpiper, in Antennas and Propagation Society International Symposium (APSURSI), IEEE., 2012, p. 1

20. N. Zhu, R.W. Ziolkowski, Appl. Phys. Lett. 101, 024107 (2012)

21. D.J. Gregoire, C.R. White, J.S. Colburn, IEEE Antennas Wirel. Propag. Lett. 10, 1586 (2011)

22. S.A. Tretyakov, Analytical modeling in applied electromagnetics (Artech House, 2003)

23. R.C. Hansen, M. Burke, Microw. Opt. Technol. Lett. 26, 75 (2000)

24. M.J. Freire, R. Marques, L. Jelinek, Appl. Phys. Lett. 93, 231108 (2008) 
25. H. Mosallaei, K. Sarabandi, IEEE Antennas Wirel. Propag. Lett. 4, 5 (2005)

26. A.M. Nicolson, G.F. Ross, IEEE Trans. Instrum. Meas. 19, $377(1970)$

27. W.B. Weir, Proc. IEEE 62, 33 (1974)

28. C.R. Simovski, Opt. Spectrosc. 107, 726 (2009)

29. B.A. Munk, Frequency Selective Surfaces: theory and design (John Wiley and Sons, 2005)

30. S.R. Best, D.L. Hanna, IEEE Antennas Propag. Mag. 50, 52 (2008)

31. Y. Fan, Y. Rahmat-Samii, IEEE Trans. Antennas Propag. 51, 2691 (2003)

32. N. Engheta, in Antennas and Propagation Society International Symposium, 2002 (IEEE, 2002), p. 392

33. J. Lončar, D. Muha, S. Hrabar, in 2013: IEEE International Symposium on Antennas and Propagation \& USNC/URSI National Radio Science Meeting 2015, p. 73

34. T. Kaneko, Y. Horii, in: 2013 Asia-Pacific Microwave Conference Proceedings (APMC), 2013, p. 1194

35. A.M. Elfrgani, R.G. Rojas, in: IEEE MTT-S International Microwave Symposium, 2015, p. 1

36. J. Loncar, S. Hrabar, D. Muha, IEEE Trans. Antennas Propag. 65, 390 (2017)

37. R.J. Baker, CMOS: circuit design, layout, and simulation (John Wiley and Sons, 2008)

38. J.G. Linvill, Proc. IRE. 41, 725 (1953), DOI:10.1109/ JRPROC.1953.274251

39. G. Fu, S. Sonkusale, Appl. Phys. Lett. 106, 203504 (2015)
40. M.M. Jacob, J. Long, D.F. Sievenpiper, IEEE Trans. Antennas Propag. 62, 6081 (2014)

41. J. Long, D.F. Sievenpiper, IEEE Trans. Antennas Propag. 64, 3003 (2016)

42. S. Saadat et al., IEEE Microw. Wirel. Compon. Lett. 25, 118 (2015)

43. Y. Chen, K. Mouthaan, IEEE Trans. Circuits Syst. I: reg. papers. 57, 2609 (2010)

44. B.D.H. Tellegen, Philips Res. Rep. 3, 81 (1948)

45. E.H. Kopp, Proc IEEE. 53, 2125 (1965)

46. F. Yuan, CMOS Active Inductors and Transformers: Principle, Implementation and Applications (Springer, 2008)

47. C.R. White, J.W. May, J.S. Colburn, IEEE Microw. Wirel. Compon. Lett. 22, 35 (2012)

48. A.M. Elfrgani, R.G. Rojas, in: Antennas and Propagation Society International Symposium (APSURSI), 2014 IEEE. (2014), p. 464

49. E. Ugarte-Muñoz, S. Hrabar, D. Segovia-Vargas, in: Proceedings of the 5th European Conference on Antennas and Propagation (EUCAP). (2011), p. 2059

50. E. Ugarte-Munoz et al., IEEE Trans. Antennas Propag. 60, 3490 (2012)

51. N. Ayllon et al., IEEE Trans. Microw. Theor. Tech. 59, 2073 (2011)

52. E. Avignon-Meseldzija, et al., in New Circuits and Systems Conference (NEWCAS), 2015 IEEE 13th International. (2015), p. 1

Cite this article as: Emilie Avignon-Meseldzija, Thomas Lepetit, Pietro Maris Ferreira, Fabrice Boust, Negative inductance circuits for metamaterial bandwidth enhancement, EPJ Appl. Metamat. 2017, 4, 11 